\title{
INITIAL COEFFICIENT BOUNDS FOR A GENERAL CLASS OF BI-UNIVALENT FUNCTIONS
}

\author{
H. ORHAN*, N. MAGESH, AND V.K.BALAJI
}

\begin{abstract}
Inspired by the recent works of Srivastava et al. [14, Frasin and Aouf [6] and others $1,1,5,7,17,18$, we propose to investigate the coefficient estimates for a general class of analytic and bi-univalent functions. Also, we obtain estimates on the coefficients $\left|a_{2}\right|$ and $\left|a_{3}\right|$ for functions in this new class. Some interesting remarks, corollaries and applications of the results presented here are also discussed.
\end{abstract}

\section{INTRODUCTION}

Let $\mathcal{A}$ denote the class of functions of the form

$$
f(z)=z+\sum_{n=2}^{\infty} a_{n} z^{n}
$$

which are analytic in the open unit disk $\mathbb{U}=\{z: z \in \mathbb{C}$ and $|z|<1\}$. Further, by $\mathcal{S}$ we shall denote the class of all functions in $\mathcal{A}$ which are univalent in $\mathbb{U}$.

For two functions $f$ and $g$, analytic in $\mathbb{U}$, we say that the function $f(z)$ is subordinate to $g(z)$ in $\mathbb{U}$, and write

$$
f(z) \prec g(z), \quad z \in \mathbb{U},
$$

if there exists a Schwarz function $w(z)$, analytic in $\mathbb{U}$, with

$$
w(0)=0 \quad \text { and } \quad|w(z)|<1, \quad z \in \mathbb{U},
$$

such that

$$
f(z)=g(w(z)), \quad z \in \mathbb{U} .
$$

In particular, if the function $g$ is univalent in $\mathbb{U}$, the above subordination is equivalent to

$$
f(0)=g(0) \quad \text { and } \quad f(\mathbb{U}) \subset g(\mathbb{U}) .
$$

Some of the important and well-investigated subclasses of the univalent function class $\mathcal{S}$ include (for example) the class $\mathcal{S}^{*}(\alpha)$ of starlike functions of order $\alpha$ in $\mathbb{U}$ and the class $\mathcal{K}(\alpha)$ of convex functions of order $\alpha$ in $\mathbb{U}$. By definition, we have

$$
\mathcal{S}^{*}(\alpha):=\left\{f: f \in \mathcal{S} \text { and } \Re\left(\frac{z f^{\prime}(z)}{f(z)}\right)>\alpha ; z \in \mathbb{U} ; 0 \leq \alpha<1\right\}
$$

and

$$
\mathcal{K}(\alpha):=\left\{f: f \in \mathcal{S} \text { and } \Re\left(1+\frac{z f^{\prime \prime}(z)}{f^{\prime}(z)}\right)>\alpha ; z \in \mathbb{U} ; \quad 0 \leq \alpha<1\right\} .
$$

2000 Mathematics Subject Classification. 30C45.

Key words and phrases. Analytic functions, univalent functions, bi-univalent functions, starlike and convex functions, bi-starlike and bi-convex functions.

${ }^{*}$ Corresponding author e-mail: orhanhalit607@gmail.com. 
It readily follows from the definitions (1.2) and (1.3) that

$$
f \in \mathcal{K}(\alpha) \Longleftrightarrow z f^{\prime} \in \mathcal{S}^{*}(\alpha) \text {. }
$$

Also, let $\mathcal{S}_{\mathcal{P}}^{\beta}(\alpha)$ and $\mathcal{C}_{\mathcal{P}}^{\beta}(\alpha)$ denote the subclasses of $\mathcal{S}$ consisting functions $f(z)$ which are defined, respectively by

$$
\mathcal{S}_{\mathcal{P}}^{\beta}(\alpha):=\left\{f: f \in \mathcal{S} \text { and } \Re\left(e^{i \beta} \frac{z f^{\prime}(z)}{f(z)}\right)>\alpha \cos \beta ; z \in \mathbb{U} ; \quad 0 \leq \alpha<1, \beta \in\left(-\frac{\pi}{2}, \frac{\pi}{2}\right)\right\}
$$

and

$\mathcal{C}_{\mathcal{P}}^{\beta}(\alpha):=\left\{f: f \in \mathcal{S}\right.$ and $\left.\Re\left(e^{i \beta} \frac{z\left(f^{\prime}(z)\right)^{\prime}}{f^{\prime}(z)}\right)>\alpha \cos \beta ; z \in \mathbb{U} ; \quad 0 \leq \alpha<1, \beta \in\left(-\frac{\pi}{2}, \frac{\pi}{2}\right)\right\}$.

It is easy to see that

$$
f \in \mathcal{C}_{\mathcal{P}}^{\beta}(\alpha) \Longleftrightarrow z f^{\prime} \in \mathcal{S}_{\mathcal{P}}^{\beta}(\alpha) .
$$

It is well known that every function $f \in \mathcal{S}$ has an inverse $f^{-1}$, defined by

$$
f^{-1}(f(z))=z, z \in \mathbb{U}
$$

and

where

$$
f\left(f^{-1}(w)\right)=w,|w|<r_{0}(f) ; r_{0}(f) \geq \frac{1}{4}
$$

$$
f^{-1}(w)=w-a_{2} w^{2}+\left(2 a_{2}^{2}-a_{3}\right) w^{3}-\left(5 a_{2}^{3}-5 a_{2} a_{3}+a_{4}\right) w^{4}+\ldots
$$

A function $f \in \mathcal{A}$ is said to be bi-univalent in $\mathbb{U}$ if both $f(z)$ and $f^{-1}(z)$ are univalent in $\mathbb{U}$. Let $\Sigma$ denote the class of bi-univalent functions in $\mathbb{U}$ given by (1.1). Examples of functions in the class $\Sigma$ are

$$
\frac{z}{1-z},-\log (1-z), \frac{1}{2} \log \left(\frac{1+z}{1-z}\right)
$$

and so on. However, the familiar Koebe function is not a member of $\Sigma$. Other common examples of functions in $\mathcal{S}$ such as

$$
z-\frac{z^{2}}{2} \text { and } \frac{z}{1-z^{2}}
$$

are also not members of $\Sigma$ (see [6, 14]).

In 1967, Lewin [9] investigated the bi-univalent function class $\Sigma$ and showed that $\left|a_{2}\right|<$ 1.51. On the other hand, Brannan and Clunie [2] (see also [3, 4, 15]) and Netanyahu [11] made an attempt to introduce various subclasses of the bi-univalent function class $\Sigma$ and obtained non-sharp coefficient estimates on the first two coefficients $\left|a_{2}\right|$ and $\left|a_{3}\right|$ of (1.1). But the coefficient problem for each of the following Taylor-Maclaurin coefficients $\left|a_{n}\right|(n \in \mathbb{N} \backslash\{1,2\} ; \mathbb{N}:=\{1,2,3, \cdots\})$ is still an open problem. Following Brannan and Taha [4], many researchers (see[1, 5, 6, 7, 8, 10, 13, 14, 17, 18]) have recently introduced and investigated several interesting subclasses of the bi-univalent function class $\Sigma$ and they have found non-sharp estimates on the first two Taylor-Maclaurin coefficients $\left|a_{2}\right|$ and $\left|a_{3}\right|$.

Motivated by the above mentioned works, we define the following subclass of function class $\Sigma$. 
Definition 1.1. Let $h: \mathbb{U} \rightarrow \mathbb{C}$, be a convex univalent function such that $h(0)=1$ and $h(\bar{z})=\overline{h(z)}$, for $z \in \mathbb{U}$ and $\Re(h(z))>0$. A function $f(z)$ given by (1.1) is said to be in the class $\mathcal{N} \mathcal{P}_{\Sigma}^{\mu, \lambda}(\beta, h)$ if the following conditions are satisfied:

$$
f \in \Sigma, e^{i \beta}\left((1-\lambda)\left(\frac{f(z)}{z}\right)^{\mu}+\lambda f^{\prime}(z)\left(\frac{f(z)}{z}\right)^{\mu-1}\right) \prec h(z) \cos \beta+i \sin \beta, \quad z \in \mathbb{U}
$$

and

$$
e^{i \beta}\left((1-\lambda)\left(\frac{g(w)}{w}\right)^{\mu}+\lambda g^{\prime}(w)\left(\frac{g(w)}{w}\right)^{\mu-1}\right) \prec h(w) \cos \beta+i \sin \beta, \quad w \in \mathbb{U},
$$

where $\beta \in(-\pi / 2, \pi / 2), \lambda \geq 1, \mu \geq 0$ and the function $g$ is given by

$$
g(w)=w-a_{2} w^{2}+\left(2 a_{2}^{2}-a_{3}\right) w^{3}-\left(5 a_{2}^{3}-5 a_{2} a_{3}+a_{4}\right) w^{4}+\ldots
$$

the extension of $f^{-1}$ to $\mathbb{U}$.

Remark 1.2. If we set $h(z)=\frac{1+A z}{1+B z},-1 \leq B<A \leq 1$, in the class $\mathcal{N} \mathcal{P}_{\Sigma}^{\mu, \lambda}(\beta, h)$, we have $\mathcal{N} \mathcal{P}_{\Sigma}^{\mu, \lambda}\left(\beta, \frac{1+A z}{1+B z}\right)$ and defined as

$$
f \in \Sigma, e^{i \beta}\left((1-\lambda)\left(\frac{f(z)}{z}\right)^{\mu}+\lambda f^{\prime}(z)\left(\frac{f(z)}{z}\right)^{\mu-1}\right) \prec \frac{1+A z}{1+B z} \cos \beta+i \sin \beta, \quad z \in \mathbb{U}
$$

and

$$
e^{i \beta}\left((1-\lambda)\left(\frac{g(w)}{w}\right)^{\mu}+\lambda g^{\prime}(w)\left(\frac{g(w)}{w}\right)^{\mu-1}\right) \prec \frac{1+A w}{1+B w} \cos \beta+i \sin \beta, \quad w \in \mathbb{U},
$$

where $\beta \in(-\pi / 2, \pi / 2), \lambda \geq 1, \mu \geq 0$ and the function $g$ is given by (1.9).

Remark 1.3. Taking $h(z)=\frac{1+(1-2 \alpha) z}{1-z}, 0 \leq \alpha<1$ in the class $\mathcal{N} \mathcal{P}_{\Sigma}^{\mu, \lambda}(\beta, h)$, we have $\mathcal{N} \mathcal{P}_{\Sigma}^{\mu, \lambda}(\beta, \alpha)$ and $f \in \mathcal{N} \mathcal{P}_{\Sigma}^{\mu, \lambda}(\beta, \alpha)$ if the following conditions are satisfied:

$$
f \in \Sigma, \Re\left(e^{i \beta}\left((1-\lambda)\left(\frac{f(z)}{z}\right)^{\mu}+\lambda f^{\prime}(z)\left(\frac{f(z)}{z}\right)^{\mu-1}\right)\right)>\alpha \cos \beta, 0 \leq \alpha<1 ; z \in \mathbb{U}
$$

and

$$
\Re\left(e^{i \beta}\left((1-\lambda)\left(\frac{g(w)}{w}\right)^{\mu}+\lambda g^{\prime}(w)\left(\frac{g(w)}{w}\right)^{\mu-1}\right)\right)>\alpha \cos \beta, 0 \leq \alpha<1 ; w \in \mathbb{U},
$$

where $\beta \in(-\pi / 2, \pi / 2), \lambda \geq 1, \mu \geq 0$ and the function $g$ is given by (1.9).

Remark 1.4. Taking $\lambda=1$ and $h(z)=\frac{1+(1-2 \alpha) z}{1-z}, 0 \leq \alpha<1$ in the class $\mathcal{N P}_{\Sigma}^{\mu, \lambda}(\beta, h)$, we have $\mathcal{N} \mathcal{P}_{\Sigma}^{\mu, 1}(\beta, \alpha)$ and $f \in \mathcal{N} \mathcal{P}_{\Sigma}^{\mu, 1}(\beta, \alpha)$ if the following conditions are satisfied:

$$
f \in \Sigma, \Re\left(e^{i \beta} f^{\prime}(z)\left(\frac{f(z)}{z}\right)^{\mu-1}\right)>\alpha \cos \beta, 0 \leq \alpha<1 ; \mu \geq 0 ; z \in \mathbb{U}
$$

and

$$
\Re\left(e^{i \beta} g^{\prime}(w)\left(\frac{g(w)}{w}\right)^{\mu-1}\right)>\alpha \cos \beta, 0 \leq \alpha<1 ; \mu \geq 0 ; w \in \mathbb{U},
$$

where $\beta \in(-\pi / 2, \pi / 2)$ and the function $g$ is given by (1.9) . 
Remark 1.5. Taking $\mu+1=\lambda=1$ and $h(z)=\frac{1+(1-2 \alpha) z}{1-z}, 0 \leq \alpha<1$ in the class $\mathcal{N} \mathcal{P}_{\Sigma}^{\mu, \lambda}(\beta, h)$, we have $\mathcal{N} \mathcal{P}_{\Sigma}^{0,1}(\beta, \alpha)$ and $f \in \mathcal{N P}_{\Sigma}^{0,1}(\beta, \alpha)$ if the following conditions are satisfied:

$$
f \in \Sigma, \Re\left(e^{i \beta} \frac{z f^{\prime}(z)}{f(z)}\right)>\alpha \cos \beta, 0 \leq \alpha<1 ; z \in \mathbb{U}
$$

and

$$
\Re\left(e^{i \beta} \frac{w g^{\prime}(w)}{g(w)}\right)>\alpha \cos \beta, 0 \leq \alpha<1 ; w \in \mathbb{U},
$$

where $\beta \in(-\pi / 2, \pi / 2)$ and the function $g$ is given by (1.9) .

Remark 1.6. Taking $\mu=1$ and $h(z)=\frac{1+(1-2 \alpha) z}{1-z}, 0 \leq \alpha<1$ in the class $\mathcal{N P}_{\Sigma}^{\mu, \lambda}(\beta, h)$, we have $\mathcal{N P}_{\Sigma}^{1, \lambda}(\beta, \alpha)$ and $f \in \mathcal{N P}_{\Sigma}^{1, \lambda}(\beta, \alpha)$ if the following conditions are satisfied:

$$
f \in \Sigma, \Re\left(e^{i \beta}\left((1-\lambda) \frac{f(z)}{z}+\lambda f^{\prime}(z)\right)\right)>\alpha \cos \beta, 0 \leq \alpha<1 ; \lambda \geq 1 ; z \in \mathbb{U}
$$

and

$$
\Re\left(e^{i \beta}\left((1-\lambda) \frac{g(w)}{w}+\lambda g^{\prime}(w)\right)\right)>\alpha \cos \beta, 0 \leq \alpha<1 ; \lambda \geq 1 ; w \in \mathbb{U},
$$

where $\beta \in(-\pi / 2, \pi / 2)$ and the function $g$ is given by (1.9) $)$.

Remark 1.7. Taking $\mu=\lambda=1$ and $h(z)=\frac{1+(1-2 \alpha) z}{1-z}, 0 \leq \alpha<1$ in the $\operatorname{class} \mathcal{N} \mathcal{P}_{\Sigma}^{\mu, \lambda}(\beta, h)$, we have $\mathcal{N} \mathcal{P}_{\Sigma}^{1,1}(\beta, \alpha)$ and $f \in \mathcal{N P}_{\Sigma}^{1,1}(\beta, \alpha)$ if the following conditions are satisfied:

$$
f \in \Sigma, \Re\left(e^{i \beta} f^{\prime}(z)\right)>\alpha \cos \beta, 0 \leq \alpha<1 ; z \in \mathbb{U}
$$

and

$$
\Re\left(e^{i \beta} g^{\prime}(w)\right)>\alpha \cos \beta, 0 \leq \alpha<1 ; w \in \mathbb{U},
$$

where $\beta \in(-\pi / 2, \pi / 2)$ and the function $g$ is given by (1.9).

We note that

(1) $\mathcal{N} \mathcal{P}_{\Sigma}^{1,1}(0, \alpha)=\mathcal{H}_{\Sigma}^{\alpha}($ see [14] $)$

(2) $\mathcal{N} \mathcal{P}_{\Sigma}^{1, \lambda}(0, \alpha)=\mathcal{B}_{\Sigma}(\alpha, \lambda)($ see $[6])$

(3) $\mathcal{N P}_{\Sigma}^{0,1}(0, \alpha)=\mathcal{F}_{\Sigma}(\alpha) \quad($ see $[10])$

(4) $\mathcal{N P}_{\Sigma}^{\mu, 1}(0, \alpha)=\mathcal{N}_{\Sigma}^{\mu}(\alpha) \quad($ see [13] $)$

(5) $\mathcal{N} \mathcal{P}_{\Sigma}^{\mu, \lambda}(0, \alpha)=\mathcal{N}_{\Sigma}^{\mu, \lambda}(\alpha) \quad($ see [5]).

In order to derive our main result, we have to recall here the following lemma.

Lemma 1.8. [12, 16] Let the function $\varphi(z)$ given by

$$
\varphi(z)=\sum_{n=1}^{\infty} B_{n} z^{n}, \quad z \in \mathbb{U}
$$

be convex in $\mathbb{U}$. Suppose also that the function $h(z)$ given by

$$
h(z)=\sum_{n=1}^{\infty} h_{n} z^{n}, \quad z \in \mathbb{U}
$$

is holomorphic in $\mathbb{U}$. If $h(z) \prec \varphi(z), z \in \mathbb{U}$, then $\left|h_{n}\right| \leq\left|B_{1}\right|, n \in \mathbb{N}=\{1,2,3, \ldots\}$. 
The object of the present paper is to introduce a general new subclass $\mathcal{N} \mathcal{P}_{\Sigma}^{\mu, \lambda}(\beta, h)$ of the function class $\Sigma$ and obtain estimates of the coefficients $\left|a_{2}\right|$ and $\left|a_{3}\right|$ for functions in this new class $\mathcal{N} \mathcal{P}_{\Sigma}^{\mu, \lambda}(\beta, h)$.

\section{Coefficient Bounds for the function Class $\mathcal{N} \mathcal{P}_{\Sigma}^{\mu, \lambda}(\beta, h)$}

In this section we find the estimates on the coefficients $\left|a_{2}\right|$ and $\left|a_{3}\right|$ for functions in the class $\mathcal{N} \mathcal{P}_{\Sigma}^{\mu, \lambda}(\beta, h)$.

Theorem 2.1. Let $f(z)$ given by (1.1) be in the class $\mathcal{N} \mathcal{P}_{\Sigma}^{\mu, \lambda}(\beta, h), 0 \leq \alpha<1, \lambda \geq 1$ and $\mu g e q 0$, then

$$
\left|a_{2}\right| \leq \sqrt{\frac{2\left|B_{1}\right| \cos \beta}{(1+\mu)(2 \lambda+\mu)}}
$$

and

$$
\left|a_{3}\right| \leq \frac{2\left|B_{1}\right| \cos \beta}{(2 \lambda+\mu)(1+\mu)}
$$

where $\beta \in(-\pi / 2, \pi / 2)$.

Proof. It follows from (1.7) and (1.8) that there exists $p, q \in \mathcal{P}$ such that

$$
e^{i \beta}\left((1-\lambda)\left(\frac{f(z)}{z}\right)^{\mu}+\lambda f^{\prime}(z)\left(\frac{f(z)}{z}\right)^{\mu-1}\right)=p(z) \cos \beta+i \sin \beta
$$

and

$$
e^{i \beta}\left((1-\lambda)\left(\frac{g(w)}{w}\right)^{\mu}+\lambda g^{\prime}(w)\left(\frac{g(w)}{w}\right)^{\mu-1}\right)=p(w) \cos \beta+i \sin \beta,
$$

where $p(z) \prec h(z)$ and $q(w) \prec h(w)$ have the forms

$$
p(z)=1+p_{1} z+p_{2} z^{2}+\ldots, z \in \mathbb{U}
$$

and

$$
q(z)=1+q_{1} w+q_{2} w^{2}+\ldots, w \in \mathbb{U} .
$$

Equating coefficients in (2.3) and (2.4), we get

$$
\begin{gathered}
e^{i \beta}(\lambda+\mu) a_{2}=p_{1} \cos \beta \\
e^{i \beta}\left[\frac{a_{2}^{2}}{2}(\mu-1)+a_{3}\right](2 \lambda+\mu)=p_{2} \cos \beta \\
-e^{i \beta}(\lambda+\mu) a_{2}=q_{1} \cos \beta
\end{gathered}
$$

and

$$
e^{i \beta}\left[(\mu+3) \frac{a_{2}^{2}}{2}-a_{3}\right](2 \lambda+\mu)=q_{2} \cos \beta .
$$

From (2.7) and (2.9), we get

$$
p_{1}=-q_{1}
$$

and

$$
2 e^{i \beta}(\lambda+\mu)^{2} a_{2}^{2}=\left(p_{1}^{2}+q_{1}^{2}\right) \cos ^{2} \beta .
$$

Also, from (2.8) and (2.10), we obtain

$$
a_{2}^{2}=\frac{e^{-i \beta}\left(p_{2}+q_{2}\right) \cos \beta}{(1+\mu)(2 \lambda+\mu)} .
$$


Since $p, q \in h(\mathbb{U})$, applying Lemma 1.8, we immediately have

$$
\left|p_{m}\right|=\left|\frac{p^{(m)}(0)}{m !}\right| \leq\left|B_{1}\right|, m \in \mathbb{N},
$$

and

$$
\left|q_{m}\right|=\left|\frac{q^{(m)}(0)}{m !}\right| \leq\left|B_{1}\right|, m \in \mathbb{N} .
$$

Applying (2.14), (2.15) and Lemma 1.8 for the coefficients $p_{1}, p_{2}, q_{1}$ and $q_{2}$, we readily get

$$
\left|a_{2}\right| \leq \sqrt{\frac{2\left|B_{1}\right| \cos \beta}{(1+\mu)(2 \lambda+\mu)}} .
$$

This gives the bound on $\left|a_{2}\right|$ as asserted in (2.1).

Next, in order to find the bound on $\left|a_{3}\right|$, by subtracting (2.10) from (2.8), we get

$$
2\left(a_{3}-a_{2}^{2}\right)(2 \lambda+\mu)=e^{-i \beta}\left(p_{2}-q_{2}\right) \cos \beta .
$$

It follows from (2.13) and (2.16) that

$$
a_{3}=\frac{e^{-i \beta} \cos \beta\left(p_{2}+q_{2}\right)}{(1+\mu)(2 \lambda+\mu)}+\frac{e^{-i \beta}\left(p_{2}-q_{2}\right) \cos \beta}{2(2 \lambda+\mu)} .
$$

Applying (2.14), (2.15) and Lemma 1.8 once again for the coefficients $p_{1}, p_{2}, q_{1}$ and $q_{2}$, we readily get

$$
\left|a_{3}\right| \leq \frac{2\left|B_{1}\right| \cos \beta}{(2 \lambda+\mu)(1+\mu)} .
$$

This completes the proof of Theorem 2.1.

\section{Corollaries and Consequences}

In view of Remark 1.2, if we set

$$
h(z)=\frac{1+A z}{1+B z}, \quad-1 \leq B<A \leq 1, z \in \mathbb{U}
$$

and

$$
h(z)=\frac{1+(1-2 \alpha) z}{1-z}, 0 \leq \alpha<1, z \in \mathbb{U},
$$

in Theorem 2.1, we can readily deduce Corollaries 3.1 and 3.2, respectively, which we merely state here without proof.

Corollary 3.1. Let $f(z)$ given by (1.1) be in the class $\mathcal{N} \mathcal{P}_{\Sigma}^{\mu, \lambda}\left(\beta, \frac{1+A z}{1+B z}\right)$, then

$$
\left|a_{2}\right| \leq \sqrt{\frac{2(A-B) \cos \beta}{(1+\mu)(2 \lambda+\mu)}}
$$

and

$$
\left|a_{3}\right| \leq \frac{2(A-B) \cos \beta}{(2 \lambda+\mu)(1+\mu)},
$$

where $\beta \in(-\pi / 2, \pi / 2), \mu \geq 0$ and $\lambda \geq 1$. 
Corollary 3.2. Let $f(z)$ given by (1.1) be in the class $\mathcal{N P}_{\Sigma}^{\mu, \lambda}(\beta, \alpha), 0 \leq \alpha<1, \mu \geq 0$ and $\lambda \geq 1$, then

$$
\left|a_{2}\right| \leq \sqrt{\frac{4(1-\alpha) \cos \beta}{(1+\mu)(2 \lambda+\mu)}}
$$

and

$$
\left|a_{3}\right| \leq \frac{4(1-\alpha) \cos \beta}{(2 \lambda+\mu)(1+\mu)}
$$

where $\beta \in(-\pi / 2, \pi / 2)$.

Remark 3.3. When $\beta=0$ the estimates of the coefficients $\left|a_{2}\right|$ and $\left|a_{3}\right|$ of the Corollary 3.2 are improvement of the estimates obtained in [5, Theorem 3.1].

Corollary 3.4. Let $f(z)$ given by (1.1) be in the class $\mathcal{N P}_{\Sigma}^{\mu, 1}(\beta, \alpha), 0 \leq \alpha<1$ and $\mu \geq 0$, then

$$
\left|a_{2}\right| \leq \sqrt{\frac{4(1-\alpha) \cos \beta}{(1+\mu)(2+\mu)}}
$$

and

$$
\left|a_{3}\right| \leq \frac{4(1-\alpha) \cos \beta}{(2+\mu)(1+\mu)}
$$

where $\beta \in(-\pi / 2, \pi / 2)$.

Corollary 3.5. Let $f(z)$ given by (1.1) be in the class $\mathcal{N} \mathcal{P}_{\Sigma}^{0,1}(\beta, \alpha), 0 \leq \alpha<1$, then

$$
\left|a_{2}\right| \leq \sqrt{2(1-\alpha) \cos \beta}
$$

and

where $\beta \in(-\pi / 2, \pi / 2)$.

$$
\left|a_{3}\right| \leq 2(1-\alpha) \cos \beta
$$

Remark 3.6. Taking $\beta=0$ in Corollary [3.5, the estimate (3.7) reduces to $\left|a_{2}\right|$ of [10, Corollary 3.3] and (3.8) is improvement of $\left|a_{3}\right|$ obtained in [10, Corollary 3.3].

Corollary 3.7. Let $f(z)$ given by (1.1) be in the class $\mathcal{N P}_{\Sigma}^{1, \lambda}(\beta, \alpha), 0 \leq \alpha<1$ and $\lambda \geq 1$, then

and

$$
\left|a_{2}\right| \leq \sqrt{\frac{2(1-\alpha) \cos \beta}{2 \lambda+1}}
$$

where $\beta \in(-\pi / 2, \pi / 2)$.

$$
\left|a_{3}\right| \leq \frac{2(1-\alpha) \cos \beta}{2 \lambda+1}
$$

Remark 3.8. Taking $\beta=0$ in Corollary 3.7, the inequality (3.10) improves the estimate of $\left|a_{3}\right|$ in [6, Theorem 3.2].

Corollary 3.9. Let $f(z)$ given by (1.1) be in the class $\mathcal{N} \mathcal{P}_{\Sigma}^{1,1}(\beta, \alpha), 0 \leq \alpha<1$, then

$$
\left|a_{2}\right| \leq \sqrt{\frac{2(1-\alpha) \cos \beta}{3}}
$$

and

$$
\left|a_{3}\right| \leq \frac{2(1-\alpha) \cos \beta}{3}
$$


where $\beta \in(-\pi / 2, \pi / 2)$.

Remark 3.10. For $\beta=0$ the inequality (3.12) improves the estimate $\left|a_{3}\right|$ of [14, Theorem $2]$.

\section{REFERENCES}

[1] R. M. Ali, S.K.Lee, V.Ravichandran, S.Supramanian, Coefficient estimates for bi-univalent MaMinda starlike and convex functions, Appl. Math. Lett. 25 (2012), no. 3, 344-351.

[2] D.A. Brannan, J.G. Clunie (Eds.), Aspects of contemporary complex analysis, Academic Press, London, 1980.

[3] D. A. Brannan, J. Clunie and W. E. Kirwan, Coefficient estimates for a class of starlike functions, Canad. J. Math. 22 (1970), 476-485.

[4] D. A. Brannan and T. S. Taha, On some classes of bi-univalent functions, Studia Univ. Babeş-Bolyai Math. 31 (1986), no. 2, 70-77.

[5] M.Çağlar, H.Orhan and N.Yağmur, Coefficient bounds for new subclasses of bi-univalent functions, Filomat, in press.

[6] B. A. Frasin and M. K. Aouf, New subclasses of bi-univalent functions, Appl. Math. Lett. 24 (2011), no. $9,1569-1573$.

[7] S.P.Goyal and P.Goswami, Estimate for initial Maclaurin coefficients of bi-univalent functions for a class defined by fractional derivatives, J. Egyptian Math. Soc., 20 (2012), 179-182.

[8] T. Hayami and S. Owa, Coefficient bounds for bi-univalent functions, Pan Amer. Math. J. 22 (4) (2012), 15-26.

[9] M. Lewin, On a coefficient problem for bi-univalent functions, Proc. Amer. Math. Soc. 18 (1967), $63-68$.

[10] X.-F. Li and A.-P. Wang, Two new subclasses of bi-univalent functions, Internat. Math. Forum 7 (2012), 1495-1504.

[11] E. Netanyahu, The minimal distance of the image boundary from the origin and the second coefficient of a univalent function in $|z|<1$, Arch. Rational Mech. Anal. 32 (1969), 100-112.

[12] W. Rogosinski, On the coefficients of subordinate functions, Proc. London Math. Soc. (2) 48 (1943), $48-82$.

[13] S. Sivaprasad Kumar, V.Kumar and V. Ravichandran, Estimates for the initial coefficients of biunivalent functions, Preprint.

[14] H. M. Srivastava, A. K. Mishra and P. Gochhayat, Certain subclasses of analytic and bi-univalent functions, Appl. Math. Lett. 23 (2010), no. 10, 1188-1192.

[15] T.S. Taha, Topics in univalent function theory, Ph.D. Thesis, University of London, 1981.

[16] Q.-H. Xu, H.M. Srivastava, Z. Li, A certain subclass of analytic and close-to-convex functions, Appl. Math. Lett. 24 (2011) 396-401.

[17] Q.-H. Xu, Y.-C. Gui and H. M. Srivastava, Coefficient estimates for a certain subclass of analytic and bi-univalent functions, Appl. Math. Lett. 25 (2012), no. 6, 990-994.

[18] Q.-H. Xu, H.-G. Xiao and H. M. Srivastava, A certain general subclass of analytic and bi-univalent functions and associated coefficient estimate problems, Appl. Math. Comput. 218 (2012), no. 23, $11461-11465$.

Department of Mathematics, Faculty of Science, Ataturk University, 25240 Erzurum, TURKEY

E-mail address: orhanhalit607@gmail.com; horhan@atauni.edu.tr

Post-Graduate and Research Department of Mathematics, Government Arts College For Men, Krishnagiri 635001, Tamilnadu, India,

E-mail address: nmagi_2000@yahoo.co.in

Department of Mathematics, L.N. Govt College, Ponneri, Chennai, Tamilnadu, India

E-mail address: balajilsp@yahoo.co.in 